\title{
Topische Externa zur Prävention und Therapie hypertropher Narben und Keloide: Eine Literaturrecherche
}

\author{
Topical Preparations for Prevention and Treatment of Hypertrophic Scars and \\ Keloids: A Literature Review
}

Autoren

Institute
S. Fischer ' , H. R. Ernst ${ }^{2}$, D. Drücke ${ }^{3}$, Y. Diehm¹, M. Lehnhardt ${ }^{2}$, A. Daigeler ${ }^{2}$

Die Institutsangaben sind am Ende des Beitrags gelistet

Schlüsselwörter
Wundheilung
haut
hypertrophe Narbe
keloid
Key wordse Externa
whin
kypertrophic scars
topical preparations

eingereicht 20.01.2015 akzeptiert 29.03.2015

\section{Bibliografie}

DOI http://dx.doi.org/ 10.1055/s-0035-1549919

Online-Publikation: 15.6.2015

Handchir Mikrochir Plast Chir

2015; 47: 253-267

(c) Georg Thieme Verlag KG

Stuttgart · New York

ISSN 0722-1819

Korrespondenzadresse

Prof. Dr. Adrien Daigeler

Klinik für Plastische Chirurgie

und Schwerbrandverletzte,

Handchirurgiezentrum

Operatives Referenzzentrum für

Gliedmaßentumoren

BG-Universitätsklinikum

Bergmannsheil

Buerkle-de-la-Camp-Platz 1

44789 Bochum

daigeler@hotmail.com

\section{Zusammenfassung}

$\nabla$

Hintergrund: Die Prävention und Therapie von hypertrophen Narben und Keloiden stellt eine besondere Herausforderung für den plastischen Chirurgen dar. Topische Externa sind aufgrund ihrer einfachen, nicht invasiven und schmerzfreien Anwendung eine attraktive Therapieoption. Von Seiten der Patienten kommt dabei regelmäßig die Frage nach der Wirksamkeit topischer Externa auf, deren Klärung Gegenstand der vorliegenden Arbeit ist.

Material und Methoden: Es wurde eine systematische Literaturrecherche in den Datenbanken Medline, Medline Plus, Oldmedline und Cochrane Library durchgeführt. Eingeschlossen und gegenübergestellt wurden sämtliche randomisierten kontrollierten Studien, Meta-analysen, systematische Übersichtsarbeiten, Anwendungsbeobachtungen und Fallbeispiele aus der deutschen und englischen Literatur, die sich mit im deutschsprachigen Raum zugelassenen Narbentopika befassen.

Ergebnisse: Es konnten 14 topische Externa identifiziert werden. Es existieren jedoch wenige Daten bezüglich ihrer Wirksamkeit im Vergleich untereinander, mit anderen Therapieoptionen oder unbehandelten Kontrollen. Lediglich zu 6 Präparaten existierten Studien mit hohem Evidenzgrad. Contractubex zeigt dabei in der Prävention pathologischer Narben die besten Ergebnisse.

Schlussfolgerung: Obwohl bei aktueller Datenlage Contractubex präventive Wirkung zeigt und empfohlen werden kann, sind weitere Studien notwendig, um die Evidenzlage zu stärken und vor allem auch die therapeutische Wirksamkeit topischer Externa zu belegen.

\section{Einleitung \\ $\nabla$}

Narben sind in der Regel gutartige Hautveränderungen, welche den Endzustand einer Defekthei-

\section{Abstract}

$\nabla$

Background: The treatment and prevention of hypertrophic scars and keloids represents a special challenge particularly in plastic surgery. Given their simple, non-invasive and painless application, topical preparations are an attractive therapeutic option. On the part of the patients, the efficacy in prevention of and therapy for hypertrophic scars and keloids of these topical preparations is regularly questioned. The clarification of this issue is subject of this review.

Material and Methods: A systematic literature research on the databases Medline, Medline Plus, Oldmedline and Cochrane Library was conducted. All randomised controlled trials, meta-analyses, systematic reviews, case series and case studies from the German and English literature, dealing with topical preparations approved in the German speaking area, were included.

Results: In this research, 14 topical preparations were identified. However, there is limited data regarding their efficacy as compared to each other, to other treatment options or untreated controls. Only for 6 topical preparations studies with high evidence level exist. Contractubex showed best results in the prevention of pathological scars.

Conclusion: Even though Contractubex shows preventive effects and can be recommended based on current data, further studies are needed to strengthen the body of evidence and to prove the therapeutic efficacy of topical preparations in general. 
Narbe auf das ursprüngliche Verletzungsgebiet beschränkt bleibt, dehnt sich das Keloid auch auf zuvor unverletzte Areale aus. Hypertrophe Narben und Keloide werden in allen Altersgruppen beobachtet. Jugendliche und Erwachsene im Alter zwischen 10 und 30 Jahren sind stärker betroffen, jüngere Kinder und ältere Erwachsene seltener [1-6]. Geschlechtsspezifische Unterschiede bestehen in Hinsicht auf Häufigkeit des Auftretens nicht $[1,3,4]$. Die Behandlung hypertropher Narben und Keloide umfasst ein breites Spektrum an therapeutischen Möglichkeiten. Prinzipiell wird zwischen operativen und konservativen Maßnahmen unterschieden. Die „International Clinical Recommendations on Scar Management", welche als Leitlinie für die Behandlung hypertropher Narben und Keloide gilt, empfiehlt als Therapie der Wahl eine Kombination aus Silicongelfolienapplikation und intraläsionaler Kortisongabe [7]. Als Therapien der zweiten Wahl gelten Kompressionstherapie, Lasertherapie sowie die chirurgische Revision mit unterstützender Silikonapplikation [8]. Nicht selten wird eine Kombination von verschiedenen Therapieansätzen erforderlich, um den bestmöglichen Erfolg zu erzielen $[9,10]$.

Aufgrund der komplexen pathophysiologischen Abläufe bei der Entstehung von hypertrophen Narben und Keloiden und der Einflussnahme verschiedener Faktoren, wie z.B. genetischer Disposition, Größe, Alter, Lokalisation der Narbenbildung usw., konnten sich nur wenige einheitliche Standardtherapien in der Praxis etablieren. Häufig beruht die Therapie eher auf klinischer Erfahrung als auf wissenschaftlich belegter Evidenz. Vor allem in Hinblick auf Narbensalben und -gele ist die Datenlage unklar. Die Präparate werden durch Werbung empfohlen und Ärzte werden oft mit der Frage nach deren Wirksamkeit konfrontiert. Die folgende Übersicht über die auf dem pharmazeutischen Markt vertretenen Narbenspezifika zur lokalen Behandlung und
Prophylaxe von hypertrophen Narben und Keloiden soll den behandelnden Ärzten helfen, gemäß dem aktuellen Stand der Literatur Auskunft über Wirksamkeit und Nutzen dieser Präparate zu geben.

\section{Material und Methoden}

Es wurde eine systematische Literaturrecherche mit insgesamt 96 Suchbegriffen in den Datenbanken Medline, Medline Plus, Oldmedline und Cochrane Library durchgeführt ( $\bullet$ Tab. 1). Eingeschlossen wurden randomisierte kontrollierte Studien, Metaanalysen, systematische Übersichtsarbeiten, Anwendungsbeobachtungen und Fallbeispiele. Berücksichtigt wurden alle Studien, die sich mit der Genese, Pathophysiologie, Therapie hypertropher Narben und Keloide und der speziellen Behandlung mit Narbensalben/-gelen befassen. Die Einteilung der Evidenzstärke von klinischen Studien erfolgte nach den Kriterien der Cochrane Collaboration [11]. Die Literaturrecherche erfolgte im März 2014.

\section{Ergebnisse}

Die durch die systematische Recherche identifizierten Narbenspezifika und deren Inhaltsstoffe sind der $\odot$ Tab. 2 und die in dieser Übersichtsarbeit eingeschlossenen Originalarbeiten zusammengefasst der $\bullet$ Tab. 3 zu entnehmen.

Bei Extractum Allii Cepae, welches sich in verschiedenen Präparaten wiederfindet, handelt es sich um Zwiebelextrakt mit dem Wirkstoff Quercetin. Zwiebelextrakt besitzt antiinflammatorische, bakteriostatische und die Kollagensynthese herunterregulierende Eigenschaften [12]. Heparin ist ein Gemisch aus Muco-

\begin{tabular}{|c|c|c|c|}
\hline \multicolumn{4}{|c|}{ Handelsnamen der Narbensalben } \\
\hline Mederma Care & Keli-med & Silgel & Kelofibrase \\
\hline Gorgonium & Vaseline & Aldara 5\% & \\
\hline \multicolumn{4}{|l|}{ Namen der Wirkstoffe } \\
\hline Extractum cepae & Nachtkerzenöl & Harnstoff & Acetylglucosamin \\
\hline Allium cepa & Panthenol & Urea & Fibronectin \\
\hline Zwiebelextrakt & Dexpanthenolum & Campher & Prolinlinoleat \\
\hline Allantoin & Silikon/-öl & Camphor & Bilsenkraut \\
\hline Allantoinum & Silikondioxid & Olivenöl & Oleum Hyoscyami \\
\hline Cordionin & Polysiloxan & Olive oil & Hyoscyamus \\
\hline Heparin & Kollagen & Aminosäuresol & Hyoscyami maceratum oleosum \\
\hline Heparinum & Collagena & Asiatsäure & Petrolatum \\
\hline Imiquimod & Cyanoacrylat & Retinoic acid & Retinsäure \\
\hline Vitamin A & Tretinoin & Aloe Vera & \\
\hline \multicolumn{4}{|c|}{ Spezielle Begriffe: deutsche Termina } \\
\hline Narbe/-n & hypertrophe Narbe & Keloid & Antihistaminika \\
\hline Narbensalbe/-gel/-creme & Narbenexternum & Narbenspezifikum & Narbenkosmetikum \\
\hline chirurgische Therapie & Operative Therapie & Lasertherapie & Pentoxyfyllin \\
\hline Steroid/e & Kompressions-therapie & Drucktherapie & Kryotherapie \\
\hline Silikontherapie & Strahlentherapie & 5-Fluorouracil & Bleomycin \\
\hline Methothrexat & Interferone & Imiquimod & Retinoide \\
\hline Verapamil & Botulinumtoxin & Vitamin $\mathrm{E}$ & Penicillin \\
\hline \multicolumn{4}{|l|}{ Colchicin } \\
\hline \multicolumn{4}{|c|}{ Spezielle Begriffe: englische Termina } \\
\hline scar/-s & hypertrophic scar & Keloid & therapy \\
\hline cream/gel & ointment & Study & Clinical \\
\hline surgery & excision & Laser & steroids \\
\hline compression & cryosurgery & Silicon & radiation \\
\hline
\end{tabular}

Tab. 1 Suchbegriffe. 


\begin{tabular}{|c|c|}
\hline Name & Inhaltsstoffe \\
\hline Contractubex & Extractum Allii Cepae, Heparin, Allantoin \\
\hline Mederma Care & Extractum Allii Cepae, Allantoin, Nachtkerzenöl, Panthenol \\
\hline Striatridin & Olivenöl, Cetearyloctanoat, Aminosäuresol, Ethylnicotinat \\
\hline Keli-med & $\begin{array}{l}\text { Extractum Allii Cepae, Hyoscyami maceratum oleosum, Allantoinum Heparinum natricum, } \\
\text { UV-A-Filter: avobenzonum, UV-B-Filter:3-(4-methylbenzyliden)bornan-2-onum }\end{array}$ \\
\hline Dermatix & Polysiloxan, Silikondioxid \\
\hline Gorgonium & Heparinum, Allantoinum, Dexpanthenolum \\
\hline Kelofibrase & Harnstoff, Heparin-Natrium, Campher \\
\hline Silgel & Polysiloxan \\
\hline Terproline & Asiatsäure aus Hydrocotylenextrakt, Acetylglucosamin, Glycopeptide des Fibronektins, Prolinlinoleat \\
\hline Wipescar & Cyanoacrylat \\
\hline \multicolumn{2}{|l|}{ Imiquimod } \\
\hline \multicolumn{2}{|l|}{ Vaseline } \\
\hline \multicolumn{2}{|l|}{ Vitamin A } \\
\hline Aloe Vera & \\
\hline
\end{tabular}

Tab. 2 Narbenspezifika und deren Inhaltsstoffe.

polysacchariden mit antikoagulativer und Kollagenase-fördernder Wirkung, welches die Vernetzung der Kollagenfasern zu einem gewissen Grad inhibiert [13]. Allantoin (5-ureido-hydantoin) wirkt anti-irridativ, hydratisierend und analgetisch. Es stimuliert die Zellmitose und trägt zur Entfernung von nekrotischem Material bei [14]. Nachtkerzenöl enthält Linolsäure, Linolensäure und Vitamin E [15]. Bisher konnte allerdings noch keine über den Plazeboeffekt hinausreichende Wirkung von Nachtkerzenöl festgestellt werden [16]. Panthenol ist ein Provitamin der B-Reihe. Topischen Externa wird Panthenol aufgrund des positiven Effekts auf die Elastizität der Haut und der Fähigkeit, Fibroblasten zur Proliferation anzuregen, beigemischt [17]. Topische Anwendung von Ethylnicotinat, dem Ethyl-Ester der Nikotinsäure, steigert die Proteinsynthese, vor allem von Keratin, die Synthese von Ceramiden und beschleunigt die Differenzierung von Keratinozyten. Dadurch kommt es zu einer Stabilisation der epidermalen Barrierefunktion und somit zu einem geringeren Wasserverlust der Haut [18]. Hyoscyami maceratum oleosum, das Öl des Bilsenkrautes, beinhaltet Parasympathikus-blockierende Alkaloide wie Atropin, Scopalamin und Hyoscyamin, was letztendlich einen positiven Effekt auf die Wundheilung haben soll [19]. Polysiloxan und Silikondioxid sind als Silikone Bestandteil des Silikongels Dermatix. Nach Applikation trocknet das Gel und formt eine dünne, transparente, flexible, gasdurchlässige und wasserundurchlässige Silikonfolie die an der Haut haftet. Diese physikalischen Eigenschaften sollen den Wassergehalt der Haut erhöhen und damit die Wundheilung fördern respektive die Narbenformation inhibieren [20]. Harnstoff wirkt leicht bakterizid und löst nekrotische Zellreste auf [21]. Der Zusatz von Campher in einigen Narbensalben dient der lokalen Schmerztherapie, der anti-mikrobiellen Behandlung und führt zu einer subjektiven Kühlung der Haut [22]. Asiatsäure ist wesentlich an der Regulation von Entzündungsmediatoren beteiligt und soll damit bei topischer Anwendung die Wundheilung beschleunigen [23]. Bei der Addition von Acetylglucosamin zu manchen Präparaten, besteht der theoretische Hintergrund darin, dass es zu der Gruppe Glykosaminoglycane gehört, welche einen wichtigen Bestandteil der Dermis darstellen [24]. Fibronectin, ein Glycoprotein, fördert die Adhäsion von neutrophilen Granulozyten, Monozyten, Fibroblasten und endothelialen Zellen an die Wundregion und fördert die Migration von epidermalen Zellen in das Granulationsgewebe während der Wundheilung [25]. Das topische Cyanoacrylat, welches in dem Präparat Wipescar Verwendung findet, ist eine vor Benutzung flüssige chemische Verbindung die an den meisten menschlichen Geweben und auch anderen Ma- terialien wie Latex und Stahl haftet. Scuderi et al. führen die positive Wirkung von Cyanoacrylat u.a. auf den okklusiven Effekt und antibakterielle Eigenschaften zurück [20]. Imiquimod ist ein Modulator der Immunantwort, welcher unter anderem zu Kollagenabbau führt. Durch die Reduktion des Kollagengehalts soll Imiquimod sich positiv auf die Narbenbildung auswirken [26]. Vitamin A wird physiologischer Weise benötigt, um die Integrität des Epithels aufrecht zu erhalten. Eine höhere Integrität soll den Wasserverlust reduzieren und damit Narbenentstehung positiv beeinflussen [26]. Aloe Vera ist eine tropische Pflanze, welche einen therapeutischen Effekt in einer Vielzahl an Weichteildefekten haben soll [27]. Es wird über anti-inflammatorische, immunmodulatorische, UV-protektive und die Wundheilung fördernde Eigenschaften berichtet [28]. Vaseline ist eine Creme bestehend aus einer Mischung von langkettigen aliphatischen Kohlenwasserstoffen, die in vielen Studien als Kontrolltherapie verwendet wird. Vaseline führte zu einer beschleunigten Regeneration der Hautbarriere [29]. Eine enterale Olivenölapplikation zeigte an induzierten Wunden in chronisch gestressten Mäusen u.a. eine beschleunigte Wundheilung, verbesserte Kollagenablagerung, Angiogenese und Wundkontraktion [30]. Evidenz bezüglich der topischen Applikation ist derzeit nicht vorhanden.

\section{Contractubex}

In Studien aus den Jahren 1995 und 1994 verglichen Maragakis et al. und Willital et al. die prophylaktische Anwendung von Contractubex bei postoperativen Narben nach Operationen im Brustbereich mit sonst „üblicher“ Wundbehandlung an 65 bzw. 45 Patienten. [31,32]. Der Behandlungszeitraum betrug 6 bzw. 12 Monate und die Wirkung wurde anhand der Kriterien Narbenform, Narbenfarbe, Narbenbeschaffenheit, Juckreiz und Verträglichkeit verglichen. In beiden Studien beschrieben die Autoren eine signifikant niedrigere Ausbildung pathologischer Narben und eine schnellere Abblassung des Narbenerythems durch Contractubexbehandlung. Nebenwirkungen wurden nicht beschrieben. Ho et al. führten eine Studie zur Bestimmung der Wirksamkeit von Contractubex in der Prophylaxe von Narbenbildung nach Entfernung von Tattoos mittels YAG Lasers durch [33]. Von 107 Patienten wurden 52 mit Contractubex, und 55 mit Epiderm Silikonfolie behandelt. Nach 3-monatiger Behandlungsdauer zeigte sich nach Contractubexapplikation eine signifikant geringere Narbenbildung im Vergleich zur Kontrollgruppe (11,55 vs. $23,5 \%$ ). Diese 3 Studien werden als randomisierte 


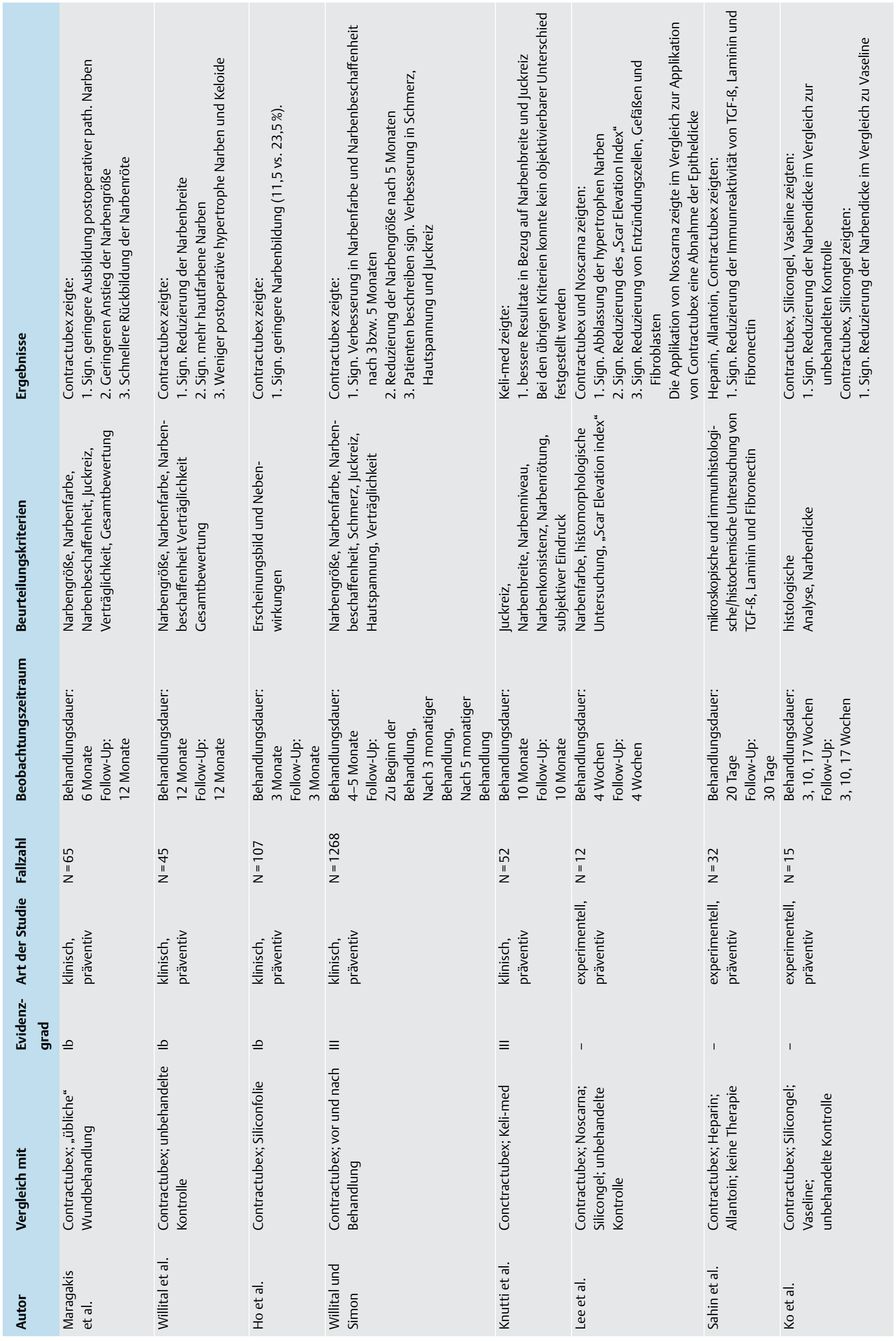




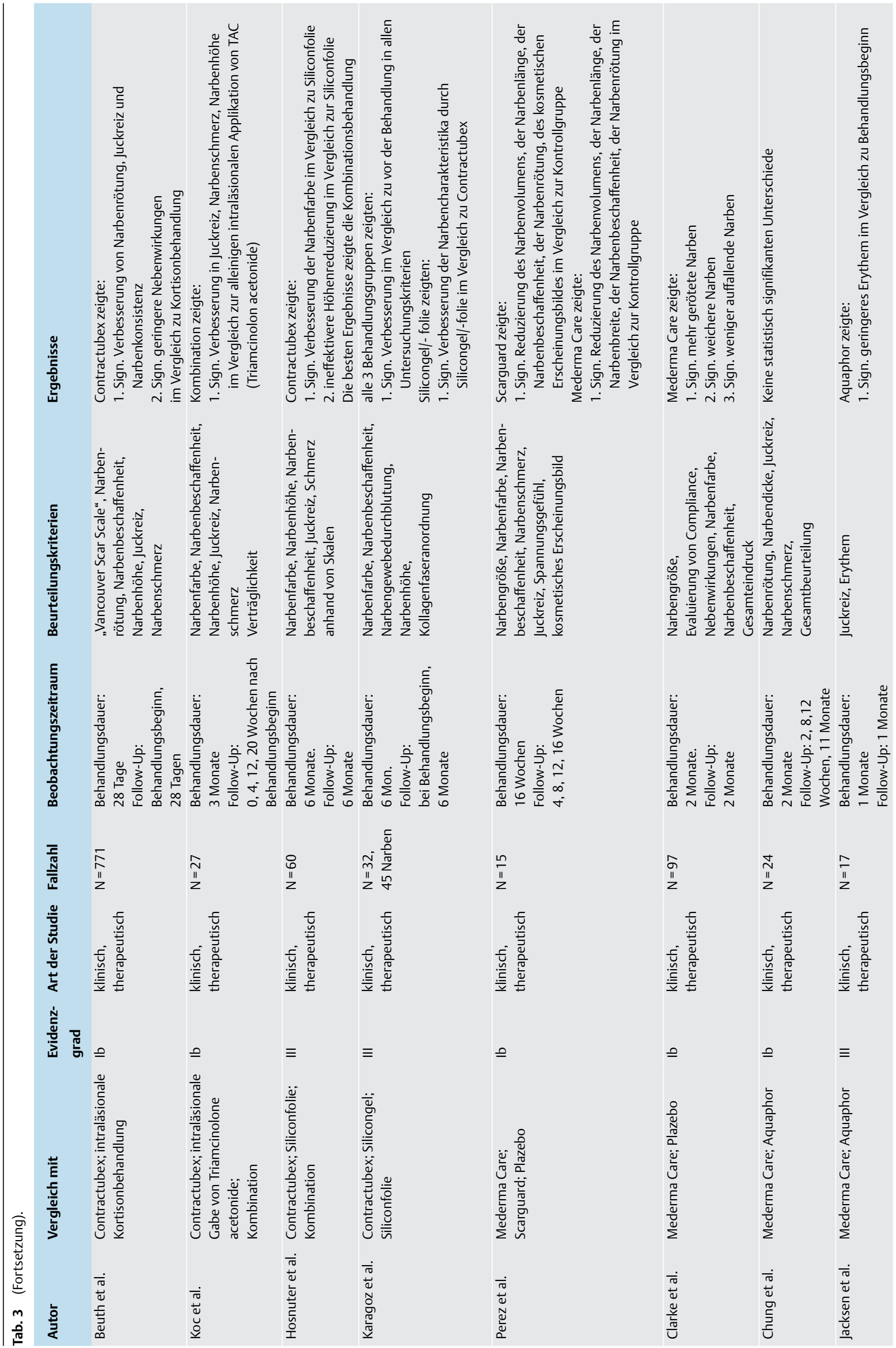




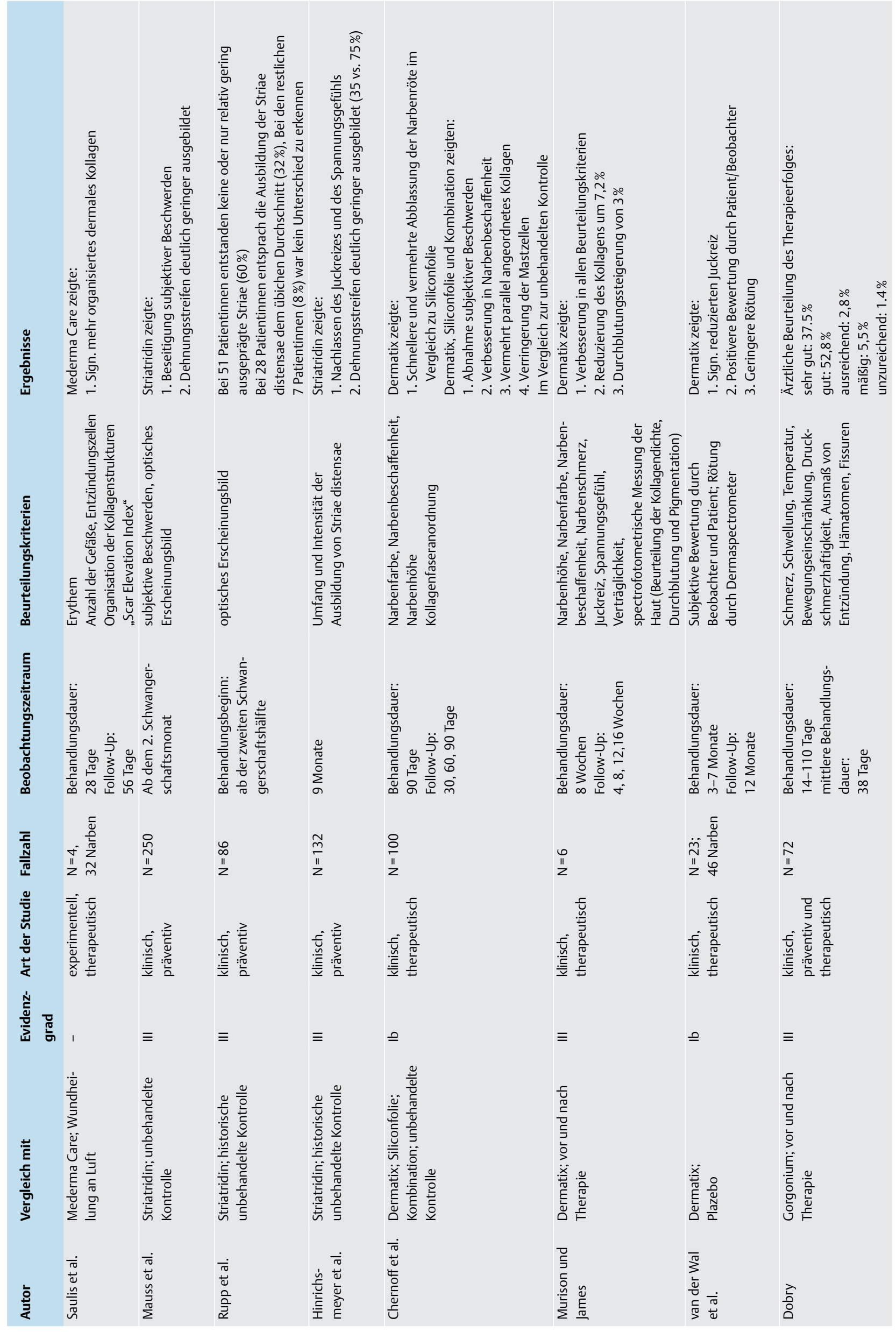



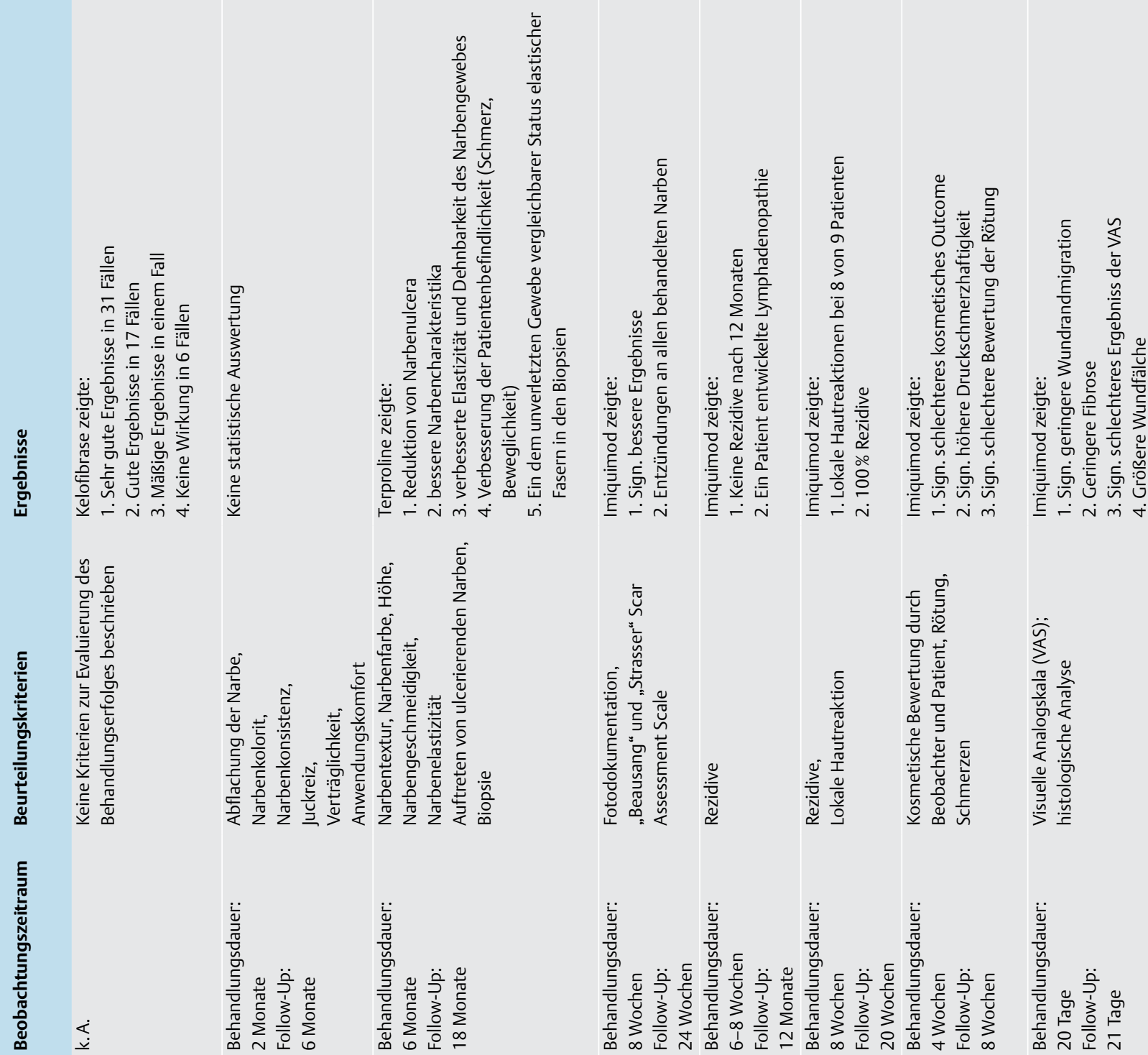

$\begin{array}{ll}\frac{\sqrt{\pi}}{\sqrt[N]{n}} & \infty \\ \frac{\infty}{11} & \frac{m}{2}\end{array}$

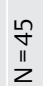

$\stackrel{\circ}{\stackrel{ }{11}}$

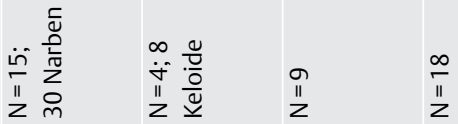

$\underset{\pi}{\pi}$

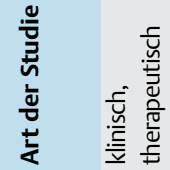

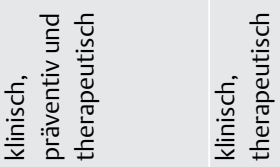

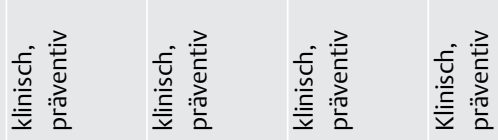

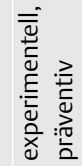

离

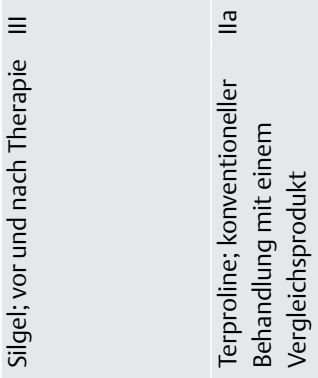

눈
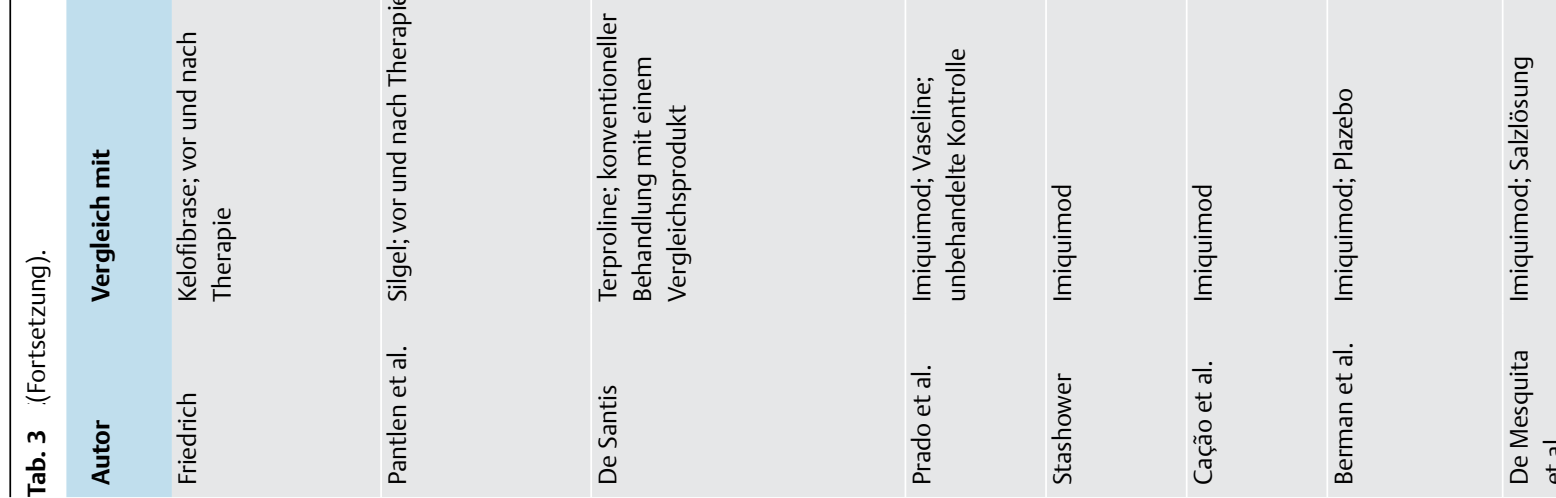

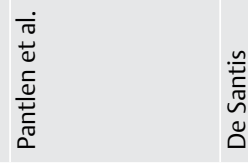

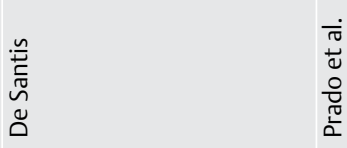

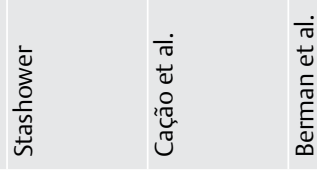

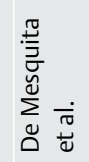



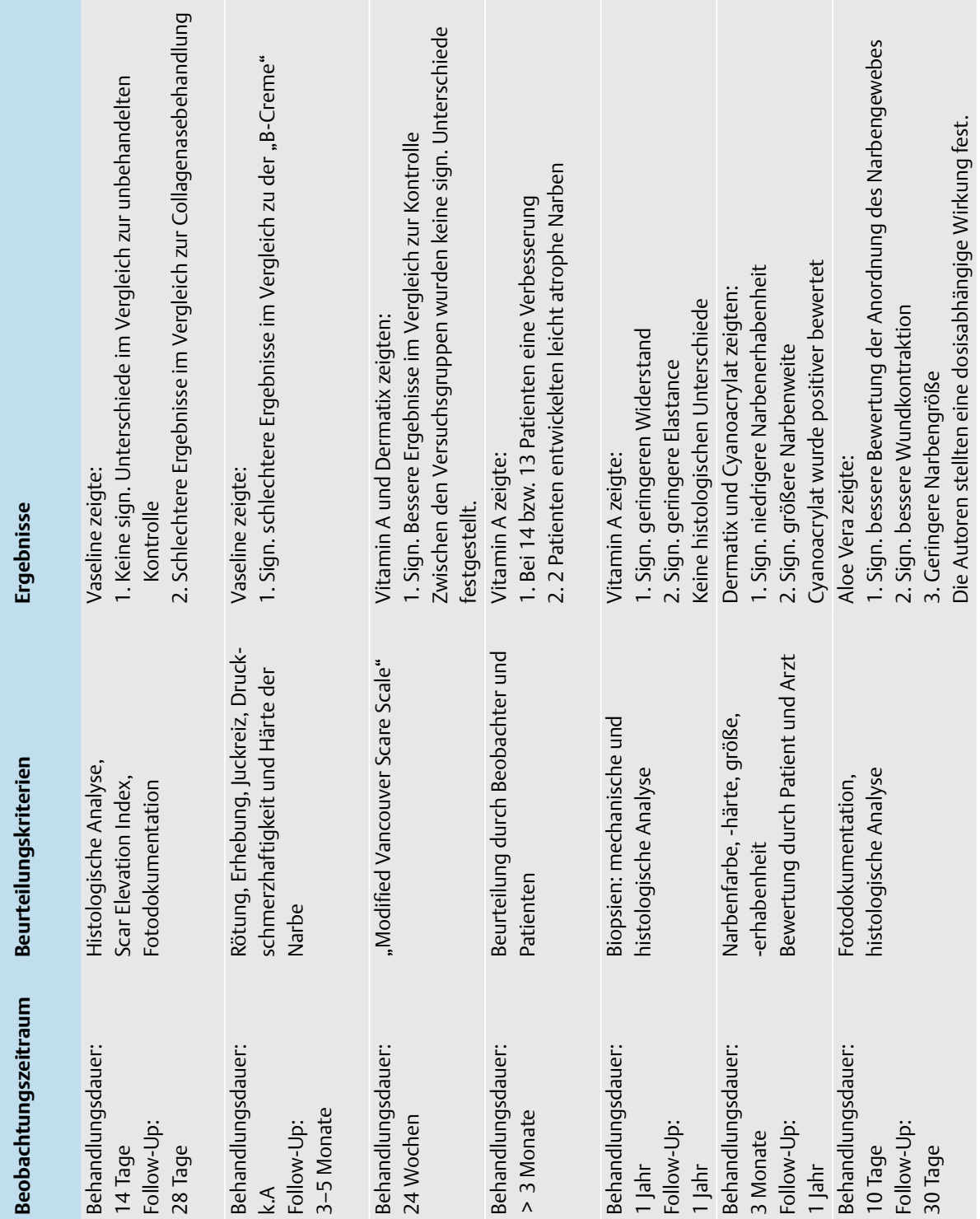

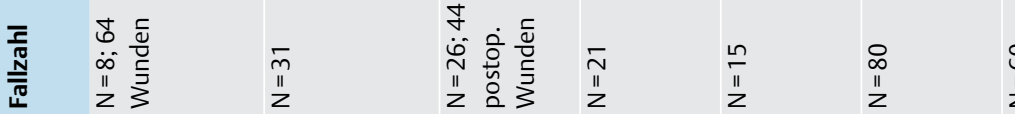

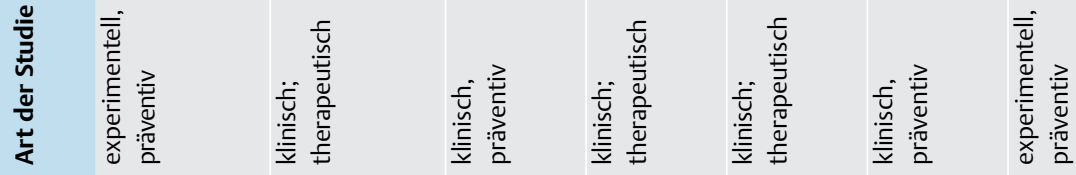

总 莺

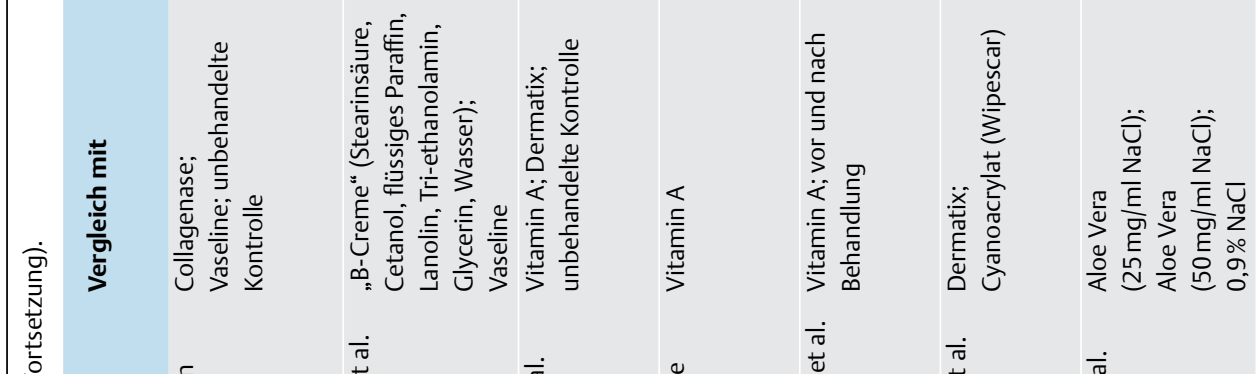

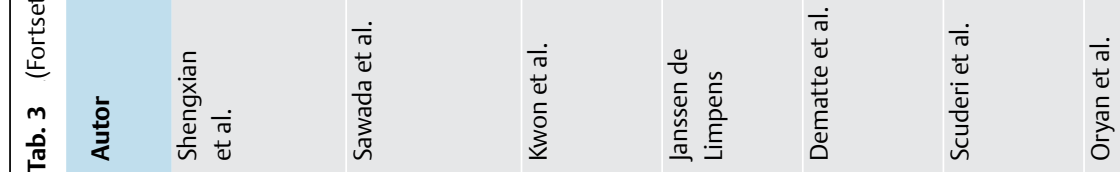


kontrollierte Studien nach den Kriterien der Cochrane Collaboration als Evidenzgrad Ib gewichtet.

Des Weiteren liegen 2 Publikationen zur prophylaktischen Contractubexbehandlung vor, die als Beobachtungsstudien den Evidenzgrad III besitzen. Willital und Simon führten eine klinische Beobachtungsstudie zur Evaluierung der Wirksamkeit von Contractubex in der Prophylaxe der überschießenden Narbenbildung an 1268 Verletzungswunden durch [9]. Bei der 2. und 3. Nachuntersuchung (3. und 5. Monat) stellten sie eine signifikante Verbesserung der Narbencharakteristika Farbe, Konsistenz und Größe sowie des Schmerzempfindens, der Hautspannung und des Juckreizes im Vergleich zum Beginn der Behandlung fest. Es gab keine Vergleichsgruppe. Knutti et al. verglichen in einer einfach verblindeten Vergleichsstudie die Wirksamkeit und Verträglichkeit von Contractubex und Keli-med bei der prophylaktischen Behandlung postoperativer Wunden nach Mammareduktionsplastiken an 52 Patienten [34]. Nach 10 Monaten stellten die Autoren einen signifikanten Wirkungsvorteil von Keli-med in Hinblick auf Narbenbreite und Juckreiz fest. Bei Contractubex kam es in 5 Fällen zu Therapieabbrüchen aufgrund von starkem Juckreiz.

3 tierexperimentelle Studien sind in der Literatur beschrieben, die über die prophylaktische Wirkung von Contractubex berichten. Lee et al. führten eine Studie an 12 Hasen mit insgesamt 24 induzierten Wunden zur Bestimmung der Wirksamkeit von Contractubex in der Wundheilung von hypertrophen Narben im Vergleich zu Noscarna (ein in Deutschland nicht zugelassenes Narbenspezifikum) durch [35]. Zur Beurteilung des Behandlungserfolges wurde eine spektrofotometrische Untersuchung der Narbenfarbe, eine histomorphologische Untersuchungen von Biopsien sowie die Bestimmung des Narbenvolumens durch den „Scar Elevation Index“ [36] verwendet. Die Behandlung mit beiden Narbenexterna zeigte eine signifikante Verringerung der pathologischen Narbenbildung. Jedoch führte Noscarna zusätzlich zu einer Reduzierung der Epitheldicke. Sahin et al. führten ein Tierexperiment an 32 Ratten durch, um die histochemische Wirkung von Contractubex, Heparin und Allantoin im Verlauf der Wundheilung zu überprüfen [37]. Die Autoren zeigten, dass durch Applikation von Contractubex eine verringerte Narbenbildung aufgrund der Reduzierung der Immunreaktivität von TGF-b, Laminin und Fibronectin hervorgerufen wird. Ko et al. untersuchten den Effekt von Contractubex, Silikongel und Vaseline auf die Dicke von Verbrennungsnarben an 15 Ratten im Vergleich zu einer Kontrollgruppe [38]. Contractubex, Silikongel und Vaseline wurden 2-mal täglich für 5 min einmassiert, während die Kontrollgruppe keine Behandlung erhielt. Alle 3 Versuchsgruppen zeigten signifikant dünnere Narben. Darüber hinaus waren Contractubex und das Silikongel der Anwendung von Vaseline signifikant überlegen.

Über die Therapie bereits bestehender hypertropher Narben und Keloide mit Contractubex existieren 2 kontrollierte randomisierte Studien mit Evidenzgrad Ib: In einer Kohortenstudie mit 771 Patienten verglichen Beuth et al. die Wirksamkeit und Sicherheit von Contractubex im Vergleich zur intraläsionalen Cortisonapplikation in der Behandlung hypertropher Narben [39]. Die Beurteilung erfolgte mittels „Vancouver Scar Scale“ [40]. Nach 28-tägiger Anwendungsdauer zeigte die Behandlung mit Contractubex signifikant bessere Ergebnisse in Bezug auf Wirksamkeit und Nebenwirkungen im Vergleich zur intraläsionalen Kortikoidapplikation. Koc et al. führten eine prospektive kontrollierte randomisierte Vergleichsstudie zur Evaluierung der Wirksamkeit von intraläsionaler Gabe von Triamcinolone acetonide und Contractubex in Kombination im Vergleich zur alleinigen Triamcinolone acetonide-Applikation durch [5]. Die Autoren beschrieben, dass die kombinierte Therapie effektiver in der Behandlung von hypertrophen Narben und Keloiden sei, als die alleinige Anwendung von Triamcinolone acetonide.

Hosnuter et al. untersuchten die Wirkung von Contractubex und Silikonfolie als alleinige und kombinierte Therapie an hypertrophen Narben und Keloiden von 72 Patienten [41]. Contractubex zeigte eine positive Wirkung in der Behandlung pathologischer Narbenbildung und eine signifikante Verbesserung im Beurteilungskriterium Narbenfarbe. Silikonfolie war effektiver bezüglich der Narbenhöhenreduzierung. Laut Autoren zeigte die Kombinationstherapie die besten Ergebnisse. In der Therapie von hypertrophen Narben, die ausschließlich nach Verbrennung entstanden sind, kam es jedoch zu divergierenden Ergebnissen. Hier zeigte sich in einer Vergleichsstudie von Karagoz et al. nach 6 monatiger Behandlung von 32 Patienten mit Contractubex, Silikongel oder Silikonfolie lediglich ein Vorteil durch die beiden letztgenannten [42].

\section{Mederma Care}

5 Publikationen berichten über die Therapie bereits bestehender hypertropher Narben und Keloide mit Mederma Care. Perez et al. führten eine prospektive kontrollierte randomisierte verblindete Studie mit Evidenzgrad Ib zur Bestimmung der Effektivität und Verträglichkeit von Mederma Care und Scarguard (ein in Deutschland nicht zugelassenes Narbenspezifikum) durch [43]. Als Kontrollgruppe diente die Applikation einer Feuchtigkeitslotion. Nach 16-wöchiger Behandlung von 15 hypertrophen Narben und Keloiden zeigte sich ein besseres Outcome durch beide Präparate. Jedoch war Scarguard gegenüber Mederma Care durch ein besseres Ergebnis des Erscheinungsbildes nach Behandlung überlegen. Clarke et al. veröffentlichten eine doppelt verblindete randomisierte kontrollierte Studie, die sich mit der Frage beschäftigte, ob Mederma Care an sich, oder die Massage bei Anwendung zu einer Verbesserung des Narbenbildes führt [44]. Untersucht wurden posttraumatische Narben von $97 \mathrm{~Pa}-$ tienten. Als Kontrolle wurde ein Plazebomittel mit gleicher Behandlungsanwendung verwendet. Nach 2-monatiger Therapiedauer stellten Clarke et al. keine signifikanten Unterschiede bei den physikalischen Messungen der Narbengröße und bei der Fotodokumentation fest. In der Plazebogruppe wurden weniger gerötete Narben als bei Mederma Care beobachtet. Ein signifikanter Unterschied zu Gunsten von Mederma-Care wurde in Bezug auf subjektive Auffälligkeit des Narbenbildes festgestellt. Chung et al. verglichen die Wirksamkeit von Mederma Care und Aquaphor (Vaseline) in der Behandlung von 24 postoperativen Wunden nach Basaliomentfernung [45]. Die Untersuchung erfolgte in einer prospektiven randomisierten doppelt verblindeten Studie der Evidenzstufe Ib. Hierbei konnten keine signifikanten Unterschiede beider Narbenexterna in Bezug auf die Narbenausbildung festgestellt werden. Jacksen et al. eruierten in einer Studie der Evidenzstufe III die Wirksamkeit von Mederma Care im Vergleich zu der Heilsalbe Aquaphor (Vaseline) an 17 postoperativen Narben [46]. Nach 1-monatiger Therapie zeigte sich kein signifikanter Unterschied zwischen den Gruppen.

Ein Tierexperiment von Saulis et al. überprüfte die Hypothese, dass eine 4-wöchige Behandlung mit Mederma Care das Narbengewebe nachweisbar weicher und flacher macht [47]. Hierbei wurden 32 hypertrophen Narben, welche an den Ohren von 4 New Zealand White Rabbits experimentell induziert wurden, untersucht. In der Kontrollgruppe verheilten die Narben ohne 
Intervention an der Luft. Anhand der Untersuchungsergebnisse stellten die Autoren keine signifikanten Unterschiede in Volumen, Gefäßzahl und Entzündungszellen fest. Allerdings wurde ein signifikanter Unterschied in der histologischen Auswertung der Kollagenorganisation festgestellt. Nach Behandlung mit Mederma Care enthielten die Narben mehr organisiertes dermales Kollagen als die Kontrollgruppe. Die Autoren vermuteten aufgrund der vermehrten Organisierung des Kollagengewebes durch Anwendung des Präparates eine Umwandlung der hypertrophen Narbe in eine reifere Form. Jedoch konnte keine signifikante Reduzierung der Narbenhypertrophie bewiesen werden.

Veröffentlichungen bezüglich der prophylaktischen Wirkung von Mederma Care existieren bis dato nicht.

\section{Striatridin}

Über die Therapie bzw. Prophylaxe von hyptertrophen Narben und Keloiden mit Striatridin gibt es keine veröffentlichten Untersuchungen. Dennoch wurden 3 Publikationen, die sich mit der Prophylaxe und Behandlung von Striae im Sinne von atrophischen Narben nach Schwangerschaft mit Striatridin befassen in diese Übersichtsarbeit eingeschlossen. Im Gegensatz zu anderen pathologischen Narbenformen kommt es bei atrophen Narben zur Narbenbildung unter Hautniveau. In einer kontrollierten nichtrandomisierten Studie, wurden 164 Schwangere während und nach der Schwangerschaft mit Striatridin behandelt und einer Kontrollgruppe von 86 Schwangeren ohne Behandlung gegenübergestellt [48]. Laut Verfasser wurden durch Striatridinanwendung subjektive Beschwerden fast völlig beseitigt. Diese Ergbenisse konnten durch 2 Anwendungsbeobachtungen, die sich mit der prophylaktischen Anwendung von Striatridin zur Verhinderung von Striae in der Schwangerschaft befassten, bestätigt werden. Hier kam es laut Autoren zu einer deutlich geringeren Ausbildung von Striae. [49,50]. Zusammenfassend ist jedoch anzumerken, dass sämtliche Studien einen geringen Evidenzgrad aufweisen und keine statistisch signifikanten Ergebnisse erzielten.

\section{Dermatix}

Chernoff et al. führten eine kontrollierte randomisierte und verblindete Studie zur Evaluierung der Wirksamkeit von Dermatix in der Behandlung von hypertrophen Narben und Keloiden durch [51]. Zum Vergleich wurden 100 Patienten in 3 Versuchsgruppen eingeteilt. Die erste Gruppe wurde mit Dermatix, die zweite mit Epiderm Silikonfolie und die dritte mit Dermatix und Epiderm Silikonfolie als Kombinationstherapie behandelt. Die Beurteilung erfolgte im Vergleich zu einer unbehandelten Kontrollgruppe. In allen 3 Behandlungsgruppen zeigte sich eine Verbesserung der Narbenbeschaffenheit. Die Anwendung von Dermatix führte zu einer schnelleren und vermehrten Abblassung der Narbenrötung im Vergleich zur Behandlung mit Silikonfolie. Murison und James führten eine Pilotstudie zur Prüfung der Wirksamkeit des Präparates an 6 Patienten mit hypertropher Narbe durch [52]. Die Beurteilung erfolgte mittels „Vancouver Scar Scale“. Nach 8-wöchiger Anwendung von Dermatix beschrieben die Autoren eine Reduzierung der Kollagendichte und Erhöhung der Durchblutung im Narbengewebe. Beide Studien wiesen keine statistisch signifikanten Ergebnisse auf. Van der Wal et al. untersuchten die Wirkung von Dermatix auf Verbrennungsnarben [53]. 23 Patienten mit jeweils 2 Narben wurden 2 Mal pro Tag an einer Narbe mit Dermatix, an der anderen mit einem Plazebo behandelt. Während des Follow-Ups von 12 Monaten wurden die Narben von den Patienten selbst, sowie von einem Beobachter bewertet. Zusätzlich wurde die Narbenrötung mit einem Dermaspectrometer gemessen. Die DermatixAnwendung führte zu positiven Bewertungen durch den Beobachter. Auf Seiten der Patienten führte Dermatix zu einer signifikanten Verringerung des Juckreizes. Die Rötung der Narben zeigte sich in der Versuchsgruppe verringert.

Weitere Studien, die Dermatix mit einem Cyanoacrylat-Präparat oder Vitamin A vergleichen, sind in den Abschnitten „Cyanoacrylat“ und „Vitamin A“ beschrieben.

\section{Gorgonium}

Dobry untersuchte in einer Anwendungsbeobachtung die Wirksamkeit und Verträglichkeit von Gorgonium in der Behandlung von hypertrophen Narben und Keloiden sowie in der Prophylaxe von frischen Operationsnarben [54]. Dobry beschrieb anhand der Ergebnisse von 72 Patienten, dass durch frühzeitige Behandlung mit dem Präparat die Entstehung keloidförmiger Narben vermieden werden kann und sich kleinere Keloide unter dieser Therapie vollständig zurückbilden können. Die Ergebnisse zeigten aufgrund des Studienaufbaus keine Signifikanz.

\section{Kelofibrase}

Friedrich untersuchte die Wirkung des Präparates Kelofibrase bei 183 Narbenbildungen unterschiedlicher Genese [55]. Die Beurteilung erfolgte mithilfe der Abstufung „sehr gut“, „gut“, „mäßig“ und „kein Erfolg“ ohne Angabe von Einteilungskriterien. Friedrich postulierte, dass durch Kelofibrase bei hypertrophen Narben und Keloiden in Abhängigkeit von der pathologisch-anatomischen Phase eine erfolgreiche Behandlung möglich ist. Die Ergebnisse zeigten, dass Keloide auf die Behandlung mit Kelofibrase schlechter ansprechen als hypertrophe Narben, wiesen jedoch keine Signifikanz auf.

\section{Silgel}

Pantlen et al. behandelten 17 frische Narben prophylaktisch und 28 bereits bestehende hypertrophe Narben verschiedener Genese [56]. Es bestand keine Randomisierung, Verblindung oder Kontrollgruppe. Die Autoren beschrieben in ihren Ergebnissen bei 36 Patienten eine Abflachung der Narbenbildung, bei 37 Patienten eine Abblassung der Narbenrötung und in 41 Fällen ein Weicherwerden der Narbenkonsistenz. Schlussfolgernd wurde festgestellt, dass Silgel in der Behandlung und Prophylaxe von hypertrophen Narben angewendet werden könne.

\section{Terproline}

Über das Narbenspezifikum Terproline wurde eine Veröffentlichung in die Übersicht eingeschlossen, die sich mit der Anwendung des Präparates bei pathologischer Narbenbildung bei verbrennungsinduzierten hypertrophen Narben befasst. De Santis untersuchte die Wirkung des Präparates an 40 Patienten [57]. Als Vergleich diente eine Kontrollgruppe mit 80 Patienten, die mit einer hydratisierenden Creme behandelt wurden. Beide Gruppen erhielten unterstützend eine Kompressionstherapie. Der Erfolg wurde anhand der Narbencharakteristika Konsistenz, Tastbarkeit und Farbe beurteilt. Jedes dieser Kriterien wurde von einem unabhängigen, verblindeten Untersucher auf einer Skala von 1 (beste) bis 5 (schlechteste) bewertet. Während in der Terproline-Gruppe Mittelwerte von 5.00, 3.85 bzw. 2.32 (nach 0, 90 und 180 Tagen) erreicht wurden, berechneten sich für die Kontrollgruppe Mittelwerte von 5.00, 4.95 und 4.98. Aussagen über statistische Signifikanzen wurden nicht angegeben. Zusätzlich 
wurden zu Behandlungsbeginn und nach Therapieende läsionale und periläsionale Biopsien entnommen.

Laut Autorangaben zeigten sich durch Terproline eine dramatische Reduktion von Narbenulzera und verbesserte Narbencharakteristika. Die Biopsieergebnisse zeigten einen dem unverletzten Gewebe vergleichbaren Status elastischer Fasern auf.

\section{Aldara $5 \%$ Imiquimod}

Prado et al. wendeten Imiquimod zur Prophylaxe von hyptertrophen Narben und Keloiden nach Brustoperationen an [58]. Insgesamt 30 Narben von 15 Patientinnen wurden mit Imiquimod oder Petrolatum Creme (Vaseline) behandelt und einer unbehandelten Kontrolle gegenübergestellt. Die Autoren berichteten über signifikante Verbesserungen des Narbenbildes durch die Therapie mit Imiquimod, jedoch traten bei diesen Narben anfangs lokale Entzündungen, Schmerzen und teilweise Blutungen auf. Stashower untersuchte die Wirkung von Imiquimod auf die Rezidivrate nach Exzision von Ohrläppchen-Keloiden an 4 Patienten mit insgesamt 8 Keloiden [59]. Die Patienten wurden einmal täglich über 6-8 Wochen behandelt. Nach 12 Monaten wurden keine Rezidive festgestellt. Ein Patient entwickelte unter der Therapie eine Lymphadenopathie und musste die Behandlung kurzzeitig unterbrechen. Cação et al. wendeten Imiquimod zur Rezidivprophylaxe nach operativer Entfernung von Keloiden am Körperstamm an [60]. 9 Patienten wurden über 8 Wochen täglich behandelt und das Follow-Up betrug 20 Wochen. Die Autoren beobachteten eine 100\% Rezidivrate und lokale Hautreaktionen bei 8 Patienten. Sie schlussfolgerten, dass Imiquimod nicht geeignet ist, um Rezidive von Keloiden am Körperstamm zu verhindern. In einer Studie mit 18 Patienten (36 postoperativen Wunden) stellten Berman et al. Imiquimod einem Plazebo gegenüber [61]. Beide Präparate wurden täglich über 4 Wochen angewendet. Imiquimod führte zu signifikant schlechter bewerteten kosmetischen Ergebnissen in Woche 8 und wies sign. schlechtere Ergebnisse in den Kategorien Rötung und Druckschmerz auf. De Mesquita et al. wendeten Imiquimod und eine Salzlösung (Kontrolle) an induzierten Brandwunden in 32 Ratten an [62]. Beide Präparate wurden 3 Mal pro Woche über 20 Tage aufgetragen. Es folgte eine histologische Auswertung und eine Analyse mittels visueller Analogskala. Imiquimod führte zu niedrigerem Gehalt an Typ-I-Kollagen und geringerer Fibrosebildung. Allerdings zeigten sich signifikant bessere Ergebnisse bei den mit Salzlösung behandelten Narben in der visuellen Bewertung und in der Größe der Wundfläche.

\section{Vaseline}

Vaseline, vor allem in Kombination mit Narbenmassage, wird häufig als Kontrollgruppe in Studien verwendet, welche die Wirkung von Narbenexterna untersuchen. Ko et al., Chung et al., Jacksen et al. und Prado et al. verwendeten Vaseline bzw. Aquaphor als Kontrolle. Die Ergebnisse dieser Studien sind den Abschnitten „Contractubex“, „Mederma Care“ und „Imiquimod“ zu entnehmen.

Es ist jedoch zu erwähnen, dass Chung et al. und Jacksen et al. Aquaphor, ein vaseline-ähnliches Mittel verwendeten. Morales-Burgos et al. verglichen Aquaphor mit normaler Petrolatum-Creme (Vaseline) und stellten fest, dass Aquaphor zu einem häufigeren Auftreten von Narbenrötung nach operativen Wunden führt [63].

Shengxian et al. nutzten 8 New Zealand white rabbits mit insgesamt 64 induzierten Narben um in einer Pilotstudie eine Collagenase-Behandlung der Narben mit der Applikation von Vase- line zu vergleichen [64]. In einem Teil der Studie stellten die Autoren fest, dass in ihrem Modell kein Unterschied des „Scar Elevation Index“ zwischen der Vaseline-Gruppe und einer unbehandelten Kontrolle bestand. Shengxian et al. stellten einen positiven Trend der Collagenase-Behandlung im Vergleich zu Vaseline und der unbehandelten Kontrollen fest.

Sawada et al. verglichen die therapeutische Wirkung einer „B-Creme“ (Stearinsäure, Cetanol, flüssiges Paraffin, Lanolin, Tri-ethanol-amin, Glycerin, Wasser) in Kombination mit einem Folienverband und Vaseline auf hypertrophe Narben und Keloide nach Verbrennungs- und Operationswunden [65]. Beurteilt wurden Rötung, Erhebung, Härte, Juckreiz und Schmerz der Narbe. Laut Autoren war Vaseline signifikant unterlegen.

\section{Vitamin A}

Kwon et al. behandelten 26 Patienten mit insgesamt 44 postoperativen Wunden mit Vitamin A oder Dermatix [66]. Obwohl beide Versuchsgruppen signifikante Verbesserungen in den Parametern Höhe, Geschmeidigkeit, Vaskularisierung und Pigmentierung gegenüber unbehandelten Kontrollen aufwiesen, kam es nach Vitamin A Applikation in 3 Fällen zu lokalem Brennen am Applikationsort. Im Vergleich der beiden Behandlungsgruppen untereinander konnten keine signifikanten Unterschiede festgestellt werden.

Janssen de Limpens wendete eine 0,05\%ige Retinsäurelösung zur Behandlung von hypertrophen Narben und Keloiden unterschiedlicher Genese bei 28 Patienten an [67]. 21 Patienten wurden in die Studie eingeschlossen. Während bei 14 Patienten Verbesserungen der Narbencharakteristika durch einen Untersucher festgestellt wurden, gaben 13 Patienten an, die Narben flacher zu empfinden. Zu dieser Studie ist jedoch anzumerken, dass viele Patienten bereits durch intraläsionale Gabe von Kenacort vorbehandelt waren.

Die therapeutische Wirkung von 0,05\%iger Tretinoinlösung (0,05\% Tretinoin [Vitamin-A-Säure], 5\% Propylenglycol, 50\% Alkohol) wurde durch Dematte et al. an nach Verbrennung zurückgebliebenen Narben im Gesicht geprüft [68]. 15 Patienten wendeten das Päparat täglich über einen Zeitraum von einem Jahr an. Verglichen wurden Biopsien vor und nach Behandlung. Die Mechanische Analyse der biopsierten Haut ergab nach Behandlung mit Retinsäure eine signifikante Abnahme des Widerstands mit Erhöhung der Nachgiebigkeit, was einer elastischeren und weicheren Haut entspricht. Histologische Unterschiede konnten nicht festgestellt werden. Nach Ende der Behandlung wiesen 3 Patienten post-inflammatorische Hyperpigmentierungen auf, ein Patient entwickelte milde Teleangiektasen und bei einem Patienten entwickelte sich ein Keloid. Bei 20\% der Patienten wurden Erytheme, Juckreiz und Trockenheit der Haut beobachtet. Auch in Kombination mit anderen Therapiemodalitäten wird Vitamin A erfolgreich bei Verbrennungsnarben und Narben anderer Genese angewandt. Bei der perkutanen Collageninduktion z.B., wird Vitamin A mindestens einen Monat präoperativ appliziert, um die dermale Kollagenbildung zu maximieren $[69,70]$.

Generell ist bei Vitamin A Präparaten anzumerken, dass die Anwendung stets mit einer potentiellen Teratogenität verbunden ist, auf die der Patient in jedem Fall hingewiesen werden muss [68].

\section{Wipescar}

Eine Phase 2 Multi Center Studie, durchgeführt von Scuderi et al., vergleicht den Effekt von Silikongel (Dermatix) und einem Cyanoacrylat-Präparat (Wipescar) auf die Narbenbildung nach 
bilateralen Brustoperationen [20]. 80 Patientinnen wurden an der Narbe einer Seite mit Wipescar und an der anderen Seite mit Dermatix über 3 Monate behandelt. Dermatix wurde 2-mal täglich und Wipescar alle 3-5 Tage angewandt. Bewertet wurden die Narbencharakteristika Farbe, Härte, Größe und Erhabenheit, jeweils im zeitlichen Verlauf der einzelnen Präparate, als auch im Vergleich beider Präparate miteinander. In beiden Behandlungsgruppen zeigte sich während der ein-jährigen Follow-Up Periode eine signifikante Abnahme der Erhabenheit bei signifikanter Zunahme der Narbenweite. Letztere war jedoch bei der Anwendung von Wipescar schwächer ausgeprägt. Die Autoren folgerten, dass Wipescar mindestens genauso positiv auf die Narbenbildung einwirkt wie Silikongele, da kein eindeutiger Vorteil bewiesen werden konnte.

\section{Aloe Vera}

Anhand der verwendeten Suchkriterien konnte lediglich eine Studie über die prophylaktische Therapie von hypertrophen Narben und Keloiden identifiziert werden. Oryan et al. induzierten in 60 Wistar-Ratten $2 \times 2 \mathrm{~cm}$ großer Wunden und behandelten diese mit Hochdosis und Niedrigdosis Aloe Vera Lösung (50 bzw. $25 \mathrm{mg}$ Aloe Vera pro $1 \mathrm{ml}$ Salzlösung) über einen Zeitraum von 10 Tagen [28]. Als Kontrolle diente Salzlösung ohne Aloe Vera Zusatz $(0,9 \% \mathrm{NaCl})$. Die Autoren beschrieben eine verbesserte Wundheilung und Epithelialisierung, sowie eine verringerte Narbengröße im Vergleich zur Kontrollgruppe, eine parallele Anordnung der Kollagenfasern und eine dosisabhängige Wirkung durch das Aloe Vera Präparat.

\section{Diskussion}

Die Übersicht zeigt, dass die Datenlage bezüglich der aufgeführten Präparate nicht eindeutig ist. Lediglich für eine geringe Anzahl an Narbensalben sind Studien mit hohem Evidenzgrad verfügbar. Die zu geringe Anzahl von Veröffentlichungen und Unterschiede in Bewertungskriterien, Behandlungsformen, Genese, Alter und Lokalisation der Narben machen einen direkten Vergleich der unterschiedlichen Narbensalben und -gele nur schwer möglich.

Auch der Vergleich zu anderen Therapiemodalitäten gestaltet sich problematisch. In der Literatur wird z. B. bei intraläsionaler Injektion mit Steroiden eine Rückbildungsrate der Narben zwischen 50 und 100\% angegeben [7,71]. Bei operativer Entfernung von Keloiden im gesunden Gewebe schwankt die Rezidivrate zwischen 45 und 100\% [10,72-74]. Diese Problematik der stark variierenden Rückbildungs- oder Rezidivrate gilt auch bei Narbensalben und -gelen. Eine erfolgreiche Rückbildung von hypertrophen Narben und Keloiden ist schwer objektivierbar und wird von subjektiven Kriterien beeinflusst. Eine Metaanalyse von Leventhal et al., welche die Therapieerfolge verschiedener Behandlungsmöglichkeiten von pathologischer Narbenbildung untersuchte, erfasste keinen signifikanten Behandlungsvorteil einer der verfügbaren Therapiemodalitäten [75]. Die Ergebnisse zeigten auch, dass über die Wirksamkeit etablierter Therapieoptionen bei pathologischer Narbenbildung nur eine geringe Anzahl von Studien veröffentlicht ist, die einen hohen Evidenzgrad besitzen. Ähnliche Problematik zeigte sich in der Therapie von hypertrophen Narben nach Verbrennungen. Zwar beschrieben Friedstat et al. in einer systematischen Übersichtsarbeit eine Tendenz zur Behandlung mit Silikongelfolien, erwähnten jedoch auch, dass diese Tendenz aufgrund des Aufbaus betreffender
Studien mit Vorsicht zu interpretieren sei [76]. Dementsprechend konnten Kim et al. in einer Vergleichsstudie von Silikongelfolien mit topischem Silikongel, welche beide auf dem koreanischen Markt erhältlich sind, keine Unterschiede bezüglich der Effektivität in der Narbenbehandlung feststellen [77]. Topisches Silikongel wurde jedoch von den Patienten in der Anwendung als angenehmer empfunden, was in Anbetracht der notwendigen Compliance ein entscheidendes Kriterium in der Auswahl der richtigen Narbentherapie darstellt. Die Autoren fassten zusammen, dass weitere Langzeitstudien mit größerer Fallzahl notwendig sind, um einen entsprechenden Vorteil beweisen zu können.

Im Vergleich zu anderen Therapiemöglichkeiten, wie der chirurgischen Therapie, der Behandlung mit Steroiden, Silikongelfolien oder der Laser- und Kryotherapie, sind die Narbensalben/-gele kostengünstiger. Die Preise für die Narbenexterna Contractubex, Keli-med, sowie Terproline bewegen sich bei einer Darreichungsmenge von $50 \mathrm{~g}$ in der gleichen Preisklasse zwischen 20-25 Euro und variieren nur geringfügig je nach Anbieter [7882]. Mederma Care und Gorgonium kosten bei $50 \mathrm{~g}$ zwischen 36 und 47 Euro [79,81], Kelofibrase je nach Anbieter zwischen 12 und 15 Euro $[78,80]$. Eine Ausnahme im unteren Preissegment stellen Striatridin mit ca. 7 Euro je $50 \mathrm{~g}$ [81] und Vaseline mit ca. 2-4 Euro je $100 \mathrm{ml}$ dar [78, 79, 81]. Die Silikongele Silgel und Dermatix liegen umgerechnet bei ca. 100-110 Euro/50 ml bzw. 80100 Euro/50 g $[78,80]$. Imiquimod liegt mit ca. 150 Euro pro 24 Beutel im oberen Preissegment [79,81]. Wipescar (Cyanoacrylat) kostet ca. 38 Euro/2 ml [20]. Für Aloe Vera ist aufgrund der starken Variabilität der Präparate und Anbieter eine konkrete Preisabschätzung nicht möglich.

Es muss jedoch berücksichtigt werden, dass die Kosten für die aufgeführten Narbenexterna meistens vom Patienten selbst, und nicht wie bei den meisten anderen Therapieoptionen von den Krankenkassen, getragen werden müssen. Umso wichtiger ist hier eine evidenzbasierte Datenlage zur Wirksamkeit dieser Präparate. Dies gilt vor allem auch für neuere Präparate, wie z.B. Silikongele kombiniert mit Dexapanthenol und einem Massageroller, welche definitiv weiterer wissenschaftlicher Bemühungen bedürfen, um deren potentiellen Nutzen darzustellen.

Ein Nachteil der externen Salbentherapie im Vergleich zu anderen Therapieoptionen ist jedoch die Behandlungsdauer über einen längeren Zeitraum. Das Anwendungsintervall variiert nach Literaturangaben und nach Empfehlungen der Hersteller stark. Eine konsequente Therapie über mehrere Monate oder länger wird jedoch in den meisten Fällen empfohlen [83-90]. Dies und die zum Teil mehrmals pro Tag notwendige Anwendung setzen eine hohe Compliance des Patienten voraus.

Narbensalben und -gele sind jedoch nicht invasiv, einfach anzuwenden und nicht schmerzhaft. Sie weisen wenige Nebenwirkungen auf und sind vom Patienten selbst durchzuführen. Es wäre demnach sinnvoll, zunächst eine schmerzlose Therapie mit Narbensalben zu wählen, bevor auf invasive Methoden, wie die oft schmerzhafte intraläsionale Kortikoidinjektion oder chirurgische Exzision, zurückgegriffen wird.

Als Resultat unserer Übersichtsarbeit kann auf Basis der vorliegenden Studienlage Contractubex für die Prophylaxe von hypertrophen Narben und Keloiden empfohlen werden. Das Präparat zeigte sowohl in klinischen Studien, als auch tierexperimentell, signifikante positive Behandlungsergebnisse. Die Dauer der Anwendung zur Prävention, z. B. nach chirurgischen Eingriffen, beläuft sich auf 6-12 Monate [31,32] und bewegt sich preislich bei zweimal täglicher Anwendung bei ca. 3-6 Euro $/ \mathrm{cm}^{2}$ Narbe. 
In Hinblick auf die Therapie bereits bestehender hypertropher Narben und Keloide kann allerdings keine direkte Empfehlung ausgesprochen werden. Zwar zeigte Contractubex auch hier in einzelnen Studien mit hohem Evidenzgrad signifikant positive Ergebnisse, jedoch bei unklarem Effekt über das Therapieende hinaus und ohne gesicherten Vorteil gegenüber anderen Therapieoptionen, wie bspw. Siliconfolien [41,42].

\section{Schlussfolgerung}

Die Gesamtheit der in der Literaturrecherche gefundenen Studien macht deutlich, dass die prophylaktische und therapeutische Behandlung von pathologischer Narbenbildung mit Narbensalben und -gelen insgesamt wenig untersucht ist. In den meisten Fällen handelt es sich bei den Publikationen um retrospektive Betrachtungen oder Anwendungsbeobachtungen mit kleiner Fallzahl und geringer Evidenz. Das Präparat Contractubex kann auf Basis der aktuellen Studienlage für die Prophylaxe hypertropher Narben und Keloide empfohlen werden. Um jedoch die bestmögliche Therapie für den Patienten zu gewährleisten, bedarf es zukünftig weiterer Studien um die Wirksamkeit der vorgestellten Narbenspezifika genauer zu prüfen und den teilweise erheblichen Preis, der von den Patienten selbst zu tragen ist, zu rechtfertigen.

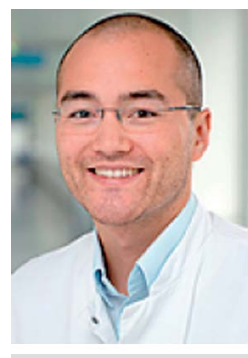

Sebastian Fischer geboren 1985 in Berlin-Kreuzberg. 2007-2011 Studium der Humanmedizin an der Ruprecht-Karls Universität Heidelberg. Promotion über die Therapie der Kapselfibrose nach Silikonimplantat basierter Brustaugmentation (Prof. G. Germann). Seit 2011 in der Facharztausbildung an der BG Klinik Ludwigshafen, Klinik für Hand-, Plastische und Rekonstruktive Chirurgie, Plastische Chirurgie der Universität Heidelberg (Direktor: Prof. U. Kneser (bis 2012 Prof. M. Lehnhardt)). Seit 2014 Postdoc im Center for Reconstructive and Restorative Surgery Research, Harvard Medical School (Prof. B. Pomahac) sowie seit 2015 am Transplant Institute, Harvard Medical School (Prof. T. B. Strom). Forschungsschwerpunkte liegen auf den immunologischen Reaktionen nach plastisch-chirurgischen Eingriffen wie der Abstoßungsreaktion nach Allo-Transplantation oder der Fremdkörperreaktion nach Insertion von Silikonimplantaten mit resultierender Kapsel- und Narbenbildung.

\section{Interessenkonflikt: Nein}

\section{Institute}

${ }^{1}$ Klinik Für Hand, Plastische und Rekonstruktive Chirurgie, Plastische Chirurgie der Universität Heidelberg, Schwerbrandverletztenzentrum, BG Klinik Ludwigshafen, Ludwigshafen

${ }^{2}$ Klinik für Plastische Chirurgie und Schwerbrandverletzte, Handchirurgiezentrum, Operatives Referenzzentrum für Gliedmaßentumoren, BG-Universitätsklinikum Bergmannsheil, Bochum

${ }^{3}$ Abteilung für Plastische Chirurgie, Klinik für Unfallchirurgie, Campus Kiel, Universitätsklinikum Schleswig-Holstein, Kiel

\section{Literatur}

1 Ketchum LD, Cohen IK, Masters FW. Hypertrophic scars and keloids. A collective review. Plastic and reconstructive surgery 1974; 53: 140-154

2 Scrimali L, Lomeo G, Tamburino $S$ et al. Laser CO2 versus radiotherapy in treatment of keloid scars. Journal of cosmetic and laser therapy: official publication of the European Society for Laser Dermatology 2012; 14: 94-97

3 Shaffer JJ, Taylor SC, Cook-Bolden F. Keloidal scars: a review with a critical look at therapeutic options. Journal of the American Academy of Dermatology 2002; 46: S63-S97

4 Hackert I, Aschoff R, Sebastian G. Keloide und ihre Therapie. Hautarzt 2003; 54: 1003-1017

5 Koc E, Arca E, Surucu B et al. An open, randomized, controlled, comparative study of the combined effect of intralesional triamcinolone acetonide and onion extract gel and intralesional triamcinolone acetonide alone in the treatment of hypertrophic scars and keloids. Dermatologic surgery: official publication for American Society for Dermatologic Surgery [et al.] 2008; 34: 1507-1514

6 Pellard S. Epidemiology, aetiology and management of abnormal scarring: a review of the literature. Journal of wound care 2006; 15: 44-48

7 Mustoe TA, Cooter RD, Gold $M H$ et al. International clinical recommendations on scar management. Plastic and reconstructive surgery 2002; 110: 560-571

8 Williams CC, De Groote S. Clinical inquiry: What treatment is best for hypertrophic scars and keloids? J Fam Pract 2011; 60: 757-758

9 Willital GH, Simon J. Efficacy of early initiation of a gel containing extractum cepae, heparin, and allantoin for scar treatment: an observational, noninterventional study of daily practice. J Drugs Dermatol 2013; 12: 38-42

10 Gauglitz GG, Pavicic T. Emerging strategies for the prevention and therapy of excessive scars. MMW Fortschr Med 2012; 154: 55-58

11 Lang B, Antes G, Bast C et al. http://www.cochrane.de (17.12.2014). Deutsches Cochrane Zentrum

12 Jenwitheesuk K, Surakunprapha P, Jenwitheesuk K et al. Role of silicone derivative plus onion extract gel in presternal hypertrophic scar protection: a prospective randomized, double blinded, controlled trial. Int Wound J 2012; 9: 397-402

13 Tarvady S, Anguli VC, Pichappa CV. Effect of heparin on wound healing. J Biosci 1987; 12: 33-40

14 Araújo LU, Grabe-Guimarães A, Mosqueira VCF et al. Profile of wound healing process induced by allantoin. Acta Cir Bras 2010; 25: 460-466

15 Kleijnen J. Evening primrose oil. BMJ: British Medical Journal 1994; 309: 824-825

16 Bamford JT, Ray S, Musekiwa A et al. Oral evening primrose oil and borage oil for eczema. The Cochrane database of systematic reviews 2013; 4: Cd004416

17 Ebner F, Heller A, Rippke $F$ et al. Topical use of dexpanthenol in skin disorders. Am J Clin Dermatol 2002; 3: 427-433

18 Gehring $W$. Nicotinic acid/niacinamide and the skin. Journal of Cosmetic Dermatology 2004; 3: 88-93

19 Pfirrmann RW, Wicki O. Method and composition for the treatment of scars. In: Google Patents 1980

20 Scuderi N, Dessy LA, Buccheri EM et al. Phase 2 cross-over multicenter trial on the efficacy and safety of topical cyanoacrylates compared with topical silicone gel in the prevention of pathologic scars. Aesthetic plastic surgery 2011; 35: 373-381

21 Holder HG, Mackay EM. The Application of Carbamide (Urea) Therapy in Wound Healing. Annals of surgery 1939; 110: 94-99

22 Raghad QM. Prophylactic effect for the sesame oil and camphor oil on the infected burn skin of mice. Tikrit Journal of Pure Science 2012; 17: 26-33

23 Sticher 0 . Triterpene einschließlich Steroide. Pharmakognosie - Phytopharmazie 2010; 9: 885-886

24 Pietramaggiori G, Yang H-J, Scherer SS et al. Effects of poly-N-acetyl glucosamine (pGlcNAc) patch on wound healing in $\mathrm{db} / \mathrm{db}$ mouse. J Trauma 2008; 64: 803-808

25 Grinnell F. Fibronectin and wound healing. J Cell Biochem 1984; 26: 107-116

26 Zurada JM, Kriegel D, Davis IC. Topical treatments for hypertrophic scars. Journal of the American Academy of Dermatology 2006; 55: 1024-1031

27 Chithra P, Sajithlal GB, Chandrakasan G. Influence of Aloe vera on collagen characteristics in healing dermal wounds in rats. Mol Cell Biochem 1998; 181: 71-76

28 Oryan A, Mohammadalipour A, Moshiri A et al. Topical Application of Aloe vera Accelerated Wound Healing, Modeling, and Remodeling: An Experimental Study With Significant Clinical Value. Annals of plastic surgery 2014; doi:10.1097/sap.0000000000000239 
29 Ghadially R, Halkier-Sorensen L, Elias PM. Effects of petrolatum on stratum corneum structure and function. Journal of the American Academy of Dermatology 1992; 26: 387-396

30 Rosa AdS, Bandeira LG, Monte-Alto-Costa A et al. Supplementation with olive oil, but not fish oil, improves cutaneous wound healing in stressed mice. Wound Repair Regen 2014; 22: 537-547

31 Maragakis M, Willital GH, Michel G et al. Possibilities of scar treatment after thoracic surgery. Drugs Exp Clin Res 1995; 21: 199-206

32 Willital GH, Heine H. Efficacy of Contractubex gel in the treatment of fresh scars after thoracic surgery in children and adolescents. Int J Clin Pharmacol Res 1994; 14: 193-202

33 Ho WS, Ying SY, Chan PC et al. Use of onion extract, heparin, allantoin gel in prevention of scarring in chinese patients having laser removal of tattoos: a prospective randomized controlled trial. Dermatologic surgery: official publication for American Society for Dermatologic Surgery [et al.] 2006; 32: 891-896

34 Knutti DA. Vergleichende therapeutische Studie zweier Narbencremen in der postoperativen Behandlung von Mammareduktionspalstiken. derm-ch 1991; 6: 14-19

35 Lee $\mathrm{DW}, \mathrm{Ku}$ SK, Cho $\mathrm{HJ}$ et al. Effects of Noscarna ${ }^{\mathrm{TM}}$ on hypertrophic scarring in the rabbit ear model: histopathological aspects. Archives of pharmacal research 2012; 35: 1999-2006

36 Morris DE, Wu L, Zhao LL et al. Acute and chronic animal models for excessive dermal scarring: quantitative studies. Plastic and reconstructive surgery 1997; 100: 674-681

37 Sahin MT, Inan S, Ozturkcan S et al. Comparison of the effects of Contractubex ${ }^{\circledR}$ gel in an experimental model of scar formation in rats: an immunohistochemical and ultrastructural study. J Drugs Dermatol 2012; 11: 74-81

38 Ko WJ, Na YC, Suh BS et al. The effects of topical agent (kelo-cote or contractubex) massage on the thickness of post-burn scar tissue formed in rats. Archives of plastic surgery 2013; 40: 697-704

39 Beuth J, Hunzelmann N, Van Leendert R et al. Safety and efficacy of local administration of contractubex to hypertrophic scars in comparison to corticosteroid treatment. Results of a multicenter, comparative epidemiological cohort study in Germany. In Vivo 2006; 20: 277-283

40 Sullivan T, Smith J, Kermode J et al. Rating the burn scar. J Burn Care Rehabil 1990; 11: 256-260

41 Hosnuter M, Payasli C, Isikdemir A et al. The effects of onion extract on hypertrophic and keloid scars. Journal of wound care $2007 ; 16$ : 251-254

42 Karagoz H, Yuksel F, Ulkur E et al. Comparison of efficacy of silicone gel, silicone gel sheeting, and topical onion extract including heparin and allantoin for the treatment of postburn hypertrophic scars. Burns 2009; 35: 1097-1103

43 Perez OA, Viera MH, Patel JK et al. A comparative study evaluating the tolerability and efficacy of two topical therapies for the treatment of keloids and hypertrophic scars. Journal of drugs in dermatology: JDD 2010; 9: 514-518

44 Clarke L, Baker B, Trahan $C$ et al. A prospective double-blinded study of Mederma skin care vs. placebo for post-traumatic scar reduction. Cosmetic Dermatol 1999; 12: 19 Y22

45 Chung VQ, Kelley L, Marra D et al. Onion Extract Gel Versus Petrolatum Emollient on New Surgical Scars: a Prospective Double-Blinded Study. Dermatologic surgery 2006; 32: 193-197

46 Jackson BA, Shelton AJ. Pilot study evaluating topical onion extract as treatment for postsurgical scars. Dermatologic surgery $1999 ; 25$ : 267-269

47 Saulis AS, Mogford JH, Mustoe TA et al. Effect of Mederma on hypertrophic scarring in the rabbit ear model. Plastic and reconstructive surgery 2002; 110: 177-183

48 Mauss HJ. Striae gravidarum-prevention as therapy. Zentralbl Gynakol 1971; 93: 1273-1278

49 Rupp Prophylaxe und Therapie der Striae distensae der Haut schwangerer Frauen. Fortschritte der Medizin 1965; 23: 951-952

50 Hinrichsmeyer $D$. Striae distensa in pregnancy, possibility and necessity of prevention. Landarzt 1966; 42: 710-713

51 Chernoff WG, Cramer H, Su-Huang S. The efficacy of topical silicone gel elastomers in the treatment of hypertrophic scars, keloid scars, and post-laser exfoliation erythema. Aesthetic plastic surgery 2007; 31: 495-500

52 Murison $M$, James $W$. Preliminary evaluation of the efficacy of dermatix silicone gel in the reduction of scar elevation and pigmentation. Journal of plastic, reconstructive \& aesthetic surgery: JPRAS 2006; 59: 437-439
53 van der Wal MBA, van Zuijlen PP, van de Ven $P$ et al. Topical silicone gel versus placebo in promoting the maturation of burn scars: a randomized controlled trial. Plastic and reconstructive surgery 2010; 126: $524-531$

54 Dobry E. Die Behandlung von frischen Narben. Therapiewoche Schweiz 12: 910-912

55 Friderich $H$. Hypertrophe Narben und Keloide Konservative Therapiemöglichkeiten. Zeitschrift für Allgemeinmedizin Heft 1985; 30: 1101-1103

56 Pantlen $P$, Tilkorn H., Schwipper V. Clinical experience with Silgel and Silgel Sheet, containing polysiloxane, in the treatment of hypertrophic scars and their prophylaxis. Forschungsbericht Fachklinik Hornheide Münster AROMANDO MEDIZIN TECHNIK 2000; www.acc.co.nz (15.01.2015)

57 de Santis P. Pilotstudie zur Wirkung von Synchroline Terproline auf verbrennungsbedingte hypertrophe Narben. http://www.synchroline. de/bilder/studien/studienliste.html (15.01.2015)

58 Prado A, Andrades $P$, Benitez $S$ et al. Scar management after breas surgery: preliminary results of a prospective, randomized, and double-blind clinical study with aldara cream $5 \%$ (imiquimod). Plastic and reconstructive surgery 2005; 115: 966-972

59 Stashower ME. Successful treatment of earlobe keloids with imiquimod after tangential shave excision. Dermatologic surgery: official publication for American Society for Dermatologic Surgery [et al.] 2006; 32: 380-386

60 Cação FM, Tanaka V, Messina MCdL. Failure of imiquimod 5\% cream to prevent recurrence of surgically excised trunk keloids. Dermatologic surgery: official publication for American Society for Dermatologic Surgery [et al.] 2009; 35: 629-633

61 Berman B, Frankel S, Villa AM et al. Double-blind, randomized, placebo-controlled, prospective study evaluating the tolerability and effectiveness of imiquimod applied to postsurgical excisions on scar cosmesis. Dermatologic surgery: official publication for American Society for Dermatologic Surgery [et al.] 2005; 31: 1399-1403

62 de Mesquita CJG, Leite JAD, Fechine FV et al. Effect of imiquimod on partial-thickness burns. Burns 2010; 36: 97-108

63 Morales-Burgos A, Loosemore MP, Goldberg LH. Postoperative wound care after dermatologic procedures: a comparison of 2 commonly used petrolatum-based ointments. J Drugs Dermatol 2013; 12: 163-164

64 Shengxian Jia M, Zhao Y, Law M, Galiano R, Mustoe TA. The Effects of Collagenase Ointment on the Prevention of Hypertrophic Scarring in a Rabbit Ear Scarring Model: A Pilot Study. WOUNDS 2011; 23: 160-165

65 Sawada Y, Sone K. Hydration and occlusion treatment for hypertrophic scars and keloids. Br J Plast Surg 1992; 45: 599-603

66 Kwon SY, Park SD, Park K. Comparative effect of topical silicone gel and topical tretinoin cream for the prevention of hypertrophic scar and keloid formation and the improvement of scars. J Eur Acad Dermatol Venereol 2014; 28: 1025-1033

67 Janssen de Limpens AM. The local treatment of hypertrophic scars and keloids with topical retinoic acid. Br J Dermatol 1980; 103: 319-323

68 Dematte MF, Gemperli R, Salles AG et al. Mechanical evaluation of the resistance and elastance of post-burn scars after topical treatment with tretinoin. Clinics (Sao Paulo) 2011; 66: 1949-1954

69 Aust MC, Fernandes D, Kolokythas $P$ et al. Percutaneous collagen induction therapy: an alternative treatment for scars, wrinkles, and skin laxity. Plastic and reconstructive surgery 2008; 121: 1421-1429

70 Aust MC, Knobloch K, Reimers $K$ et al. Percutaneous collagen induction therapy: an alternative treatment for burn scars. Burns 2010; 36: 836-843

71 Niessen FB, Spauwen PH, Schalkwijk J et al. On the nature of hypertrophic scars and keloids: a review. Plastic and reconstructive surgery 1999; 104: 1435-1458

72 Lawrence WT. In search of the optimal treatment of keloids: report of a series and a review of the literature. Annals of plastic surgery 1991; 27: 164-178

73 Cosman B, Wolff M. Correlation of keloid recurrence with completeness of local excision. A negative report. Plastic and reconstructive surgery 1972; 50: 163-166

74 Hackert I, Aschoff R, Sebastian G. Keloide und ihre Therapie. Hautarz 2003; 54: 1003-1017

75 Leventhal $D$, Furr $M$, Reiter $D$. Treatment of keloids and hypertrophic scars: a meta-analysis and review of the literature. Arch Facial Plast Surg 2006; 8: 362-368

76 Friedstat JS, Hultman CS. Hypertrophic burn scar management: what does the evidence show? A systematic review of randomized controlled trials. Annals of plastic surgery 2014; 72: S198-S201 
77 Kim S-M, Choi J-S, Lee J-H et al. Prevention of postsurgical scars: comparsion of efficacy and convenience between silicone gel sheet and topical silicone gel. J Korean Med Sci 2014; 29: (Suppl 3): S249-S253

78 Apotheke im Paunsdorf Center Kirsten Fritsch e. K. http://www.apo theke.de (15.01.2015)

79 Adler-Apotheke Aac, Untertor https://www.adlershop.ch (15.01.2015)

80 Martinus-Apotheke F-E-S, http://www.deutscheinternetapotheke.de (15.01.2015)

81 medpex Versandapotheke Stifts-Apotheke e.K. http://www.medpex.de (15.01.2015)

82 besamex LS-, https://www.besamex.de (15.01.2015)
83 Merz Pharma GmbH \& Co. Frankfurt, http://www.merz.de (15.01.2015) 84 Opfermann Arzneimittel GmbH. Im Internet: http://www.rottapharmmadaus.de (Stand: 15.01 .2015 )

85 Permamed AGP. http://www.permamed.ch (15.01.2015)

86 Advanced Bio-Technologies Inc. Silverdale WUSAViDIPGG, http://www. dermatix.de (15.01.2015)

87 Drossapharm AGB. http://www.drossapharm.ch (15.01.2015)

88 Sandoz Pharmaceuticals GmbH http://www.sandoz.de (15.01.2015)

89 AMT Aromando MGK http://www.amt-med.de (15.01.2015)

90 Dermokosmetika Synchroline. http://synchroline.de (15.01.2015) 